\title{
LV-PP-1-3
}

\section{Risk factors affecting the outcomes in pediatric liver transplantation: A real-world single-center experience}

Suk Kyun HONG, Nam-Joon Yl*, Kwangpyo HONG, Eui Soo HAN, Jeong-Moo LEE, YoungRok CHOI, Kwang-Woong LEE, Kyung-Suk SUH

Department of Surgery, Seoul National University College of Medicine, Seoul, Korea

Introduction: Despite liver transplantation (LT) being the standard treatment for pediatric end-stage liver disease, complications often persist and can adversely affect the post-transplant outcomes. This study aimed to identify the risk factors affecting the outcomes in pediatric LT patients.

Methods: Data from pediatric patients who underwent primary LT from March 1988 to December 2018 were retrospectively analyzed. Chronic liver disease was defined as an explanted liver showing fibrosis regardless of grade, cirrhosis, or any other underlying disease that may cause progressive liver injury leading to fibrosis or cirrhosis.

Results: A total of 255 pediatric patients underwent LT during the study period. Their 1-, 5-, and 10-year overall survival rates were $90.5 \%, 88.4 \%$, and $87.8 \%$. According to multivariate analysis, while liver disease without underlying chronic liver disease $(p=0.024)$ and a pediatric end-stage liver disease (PELD) score $\geq 30(p=0.036)$ were the only factors associated with worse survival, body weight $<6 \mathrm{~kg}(p=0.050)$, whole-liver DDLT compared to LDLT $(p=0.001)$, fulminant liver failure $(p=0.008)$, and postoperative hepatic artery complications $(p<0.001)$ were associated with worse graft survival. Liver disease without underlying chronic liver disease was the only factor independently associated with hepatic artery complications $(p=0.003)$.

Conclusions: Greater caution is recommended in pediatric patients with liver disease unaccompanied by underlying chronic liver disease, high PELD score, or low body weight to improve survival after LT. Hepatic artery complication was the only surgical complication affecting the graft survival outcome, especially in patients having liver disease without underlying chronic liver disease. 